López Alvarez, Marcelino; Laviana Cuetos, Margarita; Alvarez J iménez, Francisco; González Alvarez, Sergio; Fernández Doménech, María; Vera Peláez, Maria Paz

\title{
ACTIVIDAD PRODUCTIVA Y EMPLEO DE PERSONAS CON TRASTORNO MENTAL SEVERO. ALGUNAS PROPUESTAS DE ACTUACOON BASADAS EN LA INFORMACIÓN DISPONIBLE
} WORK AND EMPLOYMENT FOR PEOPLE WI TH SEVERE MENTAL DISORDERS. SOME PROPOSALS BASED ON THE AVAILABLE INFORMATION

\section{RESUMEN}

Hay un creciente interés, en el campo de la atención comunitaraia a personas con trastorno mental severo, por los temas relacionados con el empleo. Tanto en Estados Unidos como en Europa se están desarrollando a la vez nuevas iniciativas y un número creciente de investigaciones que van aportando nuevo conocimiento a este importante campo de intervención, al que, con más lentitud de la debida, vamos incorporándonos también en nuestro país.

En esa situación el artículo parte de la revisión de la información disponible sobre el tema, como base para elaborar y someter a debate algunas líneas de actuación, que recogen también los aspectos más generales de nuestra experiencia en Andalucía. A ese respecto se exponen las características generales que, en nuestra opinión, debería tener un programa destinado a facilitar actividad productiva y empleo a personas con trastorno mental severo en el contexto de nuestro país.

Palabras clave: Rehabilitación vocacional, Empleo, Integración laboral, Personas con trastorno mental severo.

\section{ABSTRACT}

There is an increassing interest, in the field of the community care for people with severe mental illness, for vocational rehabilitation and employment topics. As much in United States as in Europe there are developing, simultaneously, new initiatives and an increasing number of research that are contributing new knowledge to this important field, to which, with some delay, we are also incorporating in our country.

In this context, the paper starts with a review of the above topic, as a basis for proposing and debating some guidelines that also takes the most general aspects from our experience in Andalusia. To this respect we expose the general characteristics that, in our opinion, should have a programme to facilitate work and employment for people with severe mental illness.

Key words: Vocational Rehabilitation, Employment, Work Integration, People with Severe Mental Disorders. 


\section{INTRODUCCI ON}

El interés por el empleo de personas con enfermedades mentales graves y, especialmente, de aquellas con diagnóstico de esquizofrenia, está creciendo de manera significativa, ganando un progresivo reconocimiento profesional y ciudadano como una de las áreas a cubrir en la atención socio-sanitaria a dichas personas. Reconocimiento e interés en los que confluye una doble perspectiva (1-4), según se contemple como un derecho ciudadano o como un componente más de los programas de rehabilitación. De ahí que los programas destinados a facilitar la adquisición y mantenimiento de empleo de personas con trastorno mental severo sean, a la vez, parte de los programas de apoyo al empleo de colectivos desfavorecidos (5), y parte de los programas de atención sanitaria y social en salud mental $(6,7)$.

En realidad esa doble perspectiva, que puede ser vista como resultado de enfoques u orientaciones diferentes $(8,9)$ si bien no necesariamente contradictorios, debe ponerse en relación con los importantes cambios registrados en las últimas décadas, tanto en la consideración social de las personas con enfermedad mental como en la manera de organizar y desarrollar las distintas intervenciones que componen su atención (10-12). Así, tanto la preocupación por los derechos ciudadanos como la rehabilitación general y específicamente laboral, son componentes básicos de la llamada Salud Mental Comunitaria o Atención Comunitaria en Salud Mental, entendida como la orientación 0 «paradigma tecnológico» dominante $(11,12)$ en la atención a personas con graves problemas de salud mental.

Dicha orientación se basa en principios de salud pública, prioriza las dimensiones de funcionalidad personal y social, así como la necesidad de redes complejas de intervenciones y servicios, basadas en el trabajo en equipo y la participación, tal y como hemos señalado en otras ocasiones $(11,12)$. En concreto, con respecto a la atención a las personas con este tipo de problemas, defiende la necesidad de disponer, junto a intervenciones sanitarias de carácter biológico y psicosocial, de distintos tipos de dispositivos e intervenciones de apoyo social $(6,7)$ que tienen, a pesar de su importante diversidad, algunas no menos importantes características comunes, en términos de justificación, orientación general, principios básicos de funcionamiento y mecanismos de articulación con las intervenciones más específicamente sanitarias.

Los programas de apoyo social buscan, en coordinación pero diferenciados de los servicios sanitarios, cubrir un conjunto de necesidades básicas de las personas con discapacidades derivadas de padecer graves problemas de salud mental, incluyendo fundamentalmente alojamiento, manutención y cuidados personales, actividad y empleo, relaciones sociales, apoyo personal y tutela. Programas que tienen también en conjunto una doble justificación $(8,9,11-13)$ :

a) Por una parte pueden considerarse como complementarias de la atención sanitaria (tratamiento y rehabilitación), disponiendo de un cuerpo creciente de evi- 
dencia empírica con respecto a su utilidad para mejorar la situación clínica y, sobre todo, el funcionamiento social y el grado de satisfacción (en definitiva la calidad de vida) de las personas con trastornos psicóticos y de sus familias. $Y$ también con respecto a su contribución a disminuir el uso de recursos sanitarios, especialmente los más costosos y «traumáticos» como pueden ser los de hospitalización.

b) Y por otra, como soporte básico para asegurar el mantenimiento en la comunidad de dichas personas, eliminando barreras y mejorando su capacidad real de ejercer derechos ciudadanos básicos. Aspecto que, en las sociedades con mayor nivel de desarrollo de políticas sociales ( «estado del bienestar»), tiene cada vez más importancia y justifica por sí mismo determinadas intervenciones, aunque su contribución «medible» a mejorar parámetros estrictamente clínicos sea baja 0 no haya podido todavía comprobarse con metodología científica.

Dentro de ellos ocupan un lugar clave, en términos tanto funcionales como históricos, los específicamente destinados a facilitar distintas formas de actividad productiva y empleo, y a ellos se dedica el presente texto. A este respecto, nuestro objetivo es hacer una revisión de los principales elementos que, en nuestra opinión, caracterizan el «estado actual» del tema en sus planteamientos teóricos, incluyendo tanto sus fundamentos como las características básicas que deberían tener los programas de intervención en este área, como base para algunas propuestas a tener en cuenta para su desarrollo en nuestro país.

\section{¿QUÉ SABEMOS HOY SOBRE LA ACTI VIDAD PRODUCTIVA Y EL EMPLEO DE PERSO- NAS CON TRASTORNOS MENTALES SEVEROS}

En una primera aproximación a la creciente literatura profesional sobre el tema, es necesario enfrentarse, como en muchos campos de la atención en salud mental $(11,12)$, a algunos problemas «terminológicos», que esconden también en no pocas ocasiones, dimensiones «conceptuales».

Uno de ellos tiene que ver con la definición de la población destinataria de los programas. Dejando de lado el aún no resuelto problema de los «nombres» $(14,15)$ que nos lleva a veces a verdaderos eufemismos con tal de no utilizar «palabras malditas ${ }^{1}$ es preciso delimitar claramente en cada caso si la información hace referencia a personas con enfermedad mental, a personas con trastorno mental severo 0 a personas con Esquizofrenia (16). Términos con diferente grado de «extensión», entre los que tenemos que elegir también cuando hacemos planteamientos de futuro sobre estos programas. En concreto, cuando se revisa bibliografía sobre el tema, se pueden producir no pocas confusiones si no se delimita, claramente y en cada caso, a qué población específica se hace referencia con algunas afirmaciones. 
En nuestro caso, de lo que nos interesa hablar, en la inmensa mayoría de las ocasiones en que hacemos referencia a programas de integración laboral, es de personas con trastorno mental severo, ya que, aunque haya bastantes problemas de «ajuste laboral» en personas con otro tipo de problemas mentales, no parece haber un número importante de personas con patologías de las llamadas «menores» que presenten problemas de acceso y mantenimiento en el empleo, derivados específicamente de sus problemas psíquicos o que requieran un abordaje especial en razón de los mismos.

Pero, por otra parte, dentro del colectivo de personas con trastornos mentales severos, el grupo que plantea verdaderamente el mayor número de dificultades es el de las personas con diagnóstico de esquizofrenia. De hecho, es éste el «subgrupo» más numeroso en términos cuantitativos, así como el que se ve afectado por una mayor prevalencia de problemas de empleo: en torno al $90 \%$ no tienen empleo en nuestras sociedades (17-19), y la mayoría de los que lo obtienen, suelen perderlo, como media, en no más de seis meses (20). Es también el que presenta mayor complejidad a la hora de plantearse maneras efectivas de ayudar a sus miembros a ser y mantenerse laboralmente activos $(3,16,21)$. Si bien en muchas ocasiones se analizan las dificultades de las personas con este tipo de problemas como si constituyesen un colectivo homogéneo, y aunque, con una definición estricta de trastorno mental severo $(22,23)$, sólo una parte de los diagnosticados de esquizofrenia forman parte de ese colectivo, de hecho la mayoría de los que plantean problemas de empleo pertenecen a ambos. Es por ello que, a lo largo del presente texto, haremos referencia básicamente a personas con trastorno mental severo y diagnóstico de esquizofrenia, si bien la mayoría de las afirmaciones que vamos a hacer se aplican sin grandes dificultades al conjunto de personas con trastornos mentales graves 0 «severos».

Pero, además de esa distinción, es necesario ponerse de acuerdo también en la definición de algunos términos que usamos a veces como sinónimos, a veces con diferentes sentidos no siempre coincidentes: «trabajo», «actividad», «ocupación» y «empleo». Problema que no parece que sea exclusivo de nuestro idioma (3), pero que genera entre nosotros no pocas dificultades y confusiones. Sin pretender resolver definitivamente el problema, proponemos utilizar el término «actividad» en su acepción más general, el de «trabajo» como sinónimo de «actividad productiva» (actividad organizada que da lugar a la producción de un objeto, bien o servicio, con independencia de que no se comercialice y/ 0 retribuya, 0 de que la retribución no esté legalmente regulada) y el de «empleo» como trabajo o actividad productiva que da lugar a una retribución legalmente regulada. En este contexto reservaremos el término «ocupación» (de uso en Demografía como equivalente de «profesión») para, a través de su derivado «ocupacional», caracterizar actividades más o menos productivas, pero que no dan lugar a empleo en sentido estricto, diferenciándolas además de actividades de ocio o interacción social desprovistas del componente de producción de objetos o servicios. 
1. Actividad y empleo en personas con esquizofrenia: interés y dificultades

Si consideramos, entonces, a las personas con diagnóstico de esquizofrenia, la distinción terminológica que proponemos nos ayuda a situar, en primer lugar las funciones múltiples que asume eso que llamamos «trabajo» $(16,19,24-26)$ en la vida de todos nosotros y, especialmente, en la de las personas con esquizofrenia. En efecto, de manera resumida podemos considerar su papel en tanto que:

a) Actividad productiva, que puede servir por sí misma como estímulo al desarrollo cognitivo, como elemento organizador de la vida cotidiana y como vehículo de relaciones sociales $(26,28)$, funciones todas ellas que pueden resultar de especial importancia en la vida de estas personas;

b) Actividad retribuida, que permite la autonomía económica necesaria $(29,30)$ para desempeñar un rol social activo;

c) Actividad socialmente valorada, que aumenta la autoestima a través del rol social de trabajador y del reconocimiento social que implica $(19,31,32)$, en proporción directa a la valoración social concreta del tipo de empleo ejercido (imagen de la empresa, calidad del producto, nivel del puesto, etc (33).

Multiplicidad de funciones que permite y obliga a considerar distintas modalidades de programas de actividad productiva y empleo, que incluyan diferentes combinaciones de actividad, retribución y valoración social. Que lo deseable sea la combinación de las tres no significa que no sean útiles trabajos sin retribución o empleos de menor valoración social, cuando las combinaciones óptimas no sean alcanzables y/ o sostenibles en el tiempo (26).

Por otra parte, hay suficiente evidencia acumulada en la literatura profesional $(16,21,26,34-36)$ sobre las dificultades que una gran mayoría de estas personas presentan en este campo, dificultades de naturaleza y dimensiones múltiples, variables tanto individualmente a lo largo del tiempo, como entre unas y otras personas, y, en conjunto, bastante diferentes a las de otros colectivos con dificultades de empleo.

Dichas dificultades, que se reflejan en las elevadas cifras de desempleo que como ya se ha aludido, afectan al colectivo en diferentes lugares, resultan del encadenamiento concreto de distintos factores $(24,25,37-39)$ que dependen fundamentalmente de:

a) la propia enfermedad, afectando a áreas muy diversas de la persona (cognitivas, perceptivas, afectivas, relacionales, etc.), con evoluciones muy diversas y muy a menudo impredecibles (40-42).

b) las repercusiones que la enfermedad tiene, en la historia personal de cada uno de ellos, sobre determinadas precondiciones básicas para la futura actividad laboral (37), determinando carencias educativas, ausencia de habilidades sociales y actitudes, valores y aspiraciones habitualmente inadecuadas, 
c) los efectos derivados del propio tratamiento $(25,42,43)$, incluyendo efectos secundarios de la medicación, estrategias inadecuadas de rehabilitación, interferencias temporales entre empleo y atención sanitaria, etc.; y,

d) el conjunto de barreras sociales desarrolladas a lo largo del tiempo y que incluyen (28) aspectos que van, desde el habitual efecto desincentivador de las pensiones $(17,44,45)$, a todo el complejo de actitudes articulado por el estigma social y que afecta a los propios sujetos, a su familia, a los profesionales y a otros agentes sociales como empresarios, sindicatos, compañeros de trabajo, etc. $(13,19,46,47)$.

Barreras entre las que hay que incluir el desconocimiento de las peculiaridades de este tipo de personas y que lleva a que, más allá de las buenas intenciones, determinados programas teóricamente orientados a favorecer su acceso al empleo, funcionen de nuevo como mecanismos reforzadores de la exclusión².

En conjunto, cabe afirmar que cualquier programa que pretenda incidir seriamente en el acceso y mantenimiento en el empleo de estas personas debería tener en cuenta al menos tres conceptos clave, cuyos términos van a aparecer repetidamente a lo largo del texto: la especificidad del colectivo, con relación a otras personas con problemas de empleo (35); la variabilidad individual (sincrónica y diacrónica) que lo caracteriza (48); y, la complejidad de actividades, dispositivos y programas que hay que poner en juego para intentar modificar positivamente las distintas variables que determinan sus dificultades en este área $(11,12)$.

\section{Evolución de los programas de empleo}

En estos momentos disponemos ya de una considerable perspectiva histórica con respecto a distintos tipos de programas, utilizados a lo largo de bastantes años y en conexión con el desarrollo de diferentes modelos de atención comunitaria $(28,49$ 52). Programas sobre cuyo mayor o menor grado de éxito, a la hora de facilitar el acceso y mantenimiento en el empleo de personas con este tipo de problemas, vamos disponiendo de un volumen creciente de datos sobre resultados $(2,3,16,28,35$ 37,49,50,53-56).

A este respecto, el CUADRO I pretende ofrecer un resumen de las características generales, ventajas e inconvenientes de los principales modelos utilizados hasta ahora, en distintos países desarrollados. De él se desprende que los primeros modelos "ensayados» han tenido resultados más bien discutibles y presentan aspectos negativos, tal y como se han desarrollado históricamente. Lo que no significa que algunas de las actividades derivadas de ellos no sigan teniendo utilidad, en un contexto más amplio $(28,57)$. Así, como luego veremos, algún tipo de actividad ocupacional (derivada de formas de empleo protegido) o actividades de rehabilitación vocacional (orientación, entrenamiento en búsqueda de empleo, formación profesional, empleo 
de transición, etc.), que si por sí solas son insuficientes para mantenerse en un puesto de trabajo, pueden ser necesarias dentro de un abanico amplio de programas que permitan facilitar, de manera individualizada, distintas combinaciones de actividad productiva y empleo a diferentes miembros del colectivo que nos ocupa.

Parece, sin embargo, que los resultados obtenidos, por algunos de los programas de más reciente desarrollo, permiten afirmar que la consecución y mantenimiento en un empleo, por parte de personas con trastornos de tipo esquizofrénico, es posible en una proporción considerable de los casos $(2,3,35,36,49,50,53-56)$. Por su especial interés merece la pena detenerse un poco más en los dos últimos modelos de la tabla, de los que pensamos que, pese a desarrollarse en contextos sociales bastante diferentes entre sí (el primero en Estados Unidos y el segundo en Europa), pueden extraerse principios de funcionamiento útiles para la elaboración actual de programas concretos. Pasamos pues a describir con algo más de detalle, algunas de sus más importantes características, intentando hacer hincapié en lo que pueden tener de común $(3,50,56)$, de cara a su posible inclusión en nuestras propuestas.

En primer lugar, hay que hacer referencia a los programas de «Empleo con apoyo» (36,53-55), desarrollados básicamente en USA y, en menor medida, en Canadá (58), que cuentan con bastantes experiencias consistentemente evaluadas $(2,21,35,36,53$ 55) y que, a pesar de basarse en un contexto laboral y de servicios muy diferente al nuestro $(2,51)$, muestran algunos elementos a tener en cuenta a la hora de plantearse programas en este área.

Una importante línea de investigación intenta relacionar el éxito de este tipo de programas (con resultados positivos en términos de empleo que superan en bastantes ocasiones el $50 \%$ de los participantes, manteniéndose en el tiempo, aunque en este aspecto la «evidencia» actual es menor (34) con la «fidelidad» a determinados principios específicos del modelo $(55,59)$, tomados en conjunto, tal y como se resumen en el CUADRO II. Sin embargo, como luego veremos, parece razonable contar también con elementos concretos susceptibles de integrarse en otras aproximaciones. Así, podemos retener como factores clave los siguientes: la búsqueda y mantenimiento de empleos ordinarios ${ }^{3}$, la disponibilidad de mecanismos de apoyo individualizados, flexibles y continuados en el tiempo, con especial referencia a los derivados de la interacción cotidiana con los restantes trabajadores (60-67), y la relativa utilidad de la formación previa, si se asegura la formación y el seguimiento en el propio puesto de trabajo (68).

Factores que, junto a la atención integral de base comunitaria, las políticas de no exclusión a priori y la utilización de especialistas en empleo dentro de los servicios de salud mental, parecen ser los que mayor correlación presentan con los indicadores de éxito, en distintos estudios experimentales (59). Hay que destacar el énfasis que la mayoría de los estudios hacen en el papel múltiple y positivo que juegan los aspectos «normales» del trabajo (a diferencia de contextos clínicos, 
rehabilitadores u ocupacionales) y especialmente la interacción cotidiana con personas no-enfermas sobre la base de reglas de funcionamiento comunes $(61,65-67)$.

Y, paralelamente, hay que considerar también las experiencias de creación de «Empresas sociales» $(3,13,17,33,52,56,69-71)$, en el contexto europeo, sobre la base de intentar articular lo mejor del empleo protegido (entornos específicamente adaptados y tolerantes ${ }^{4}$ ) y del empleo con apoyo (trabajo en empresas reales y con retribución de mercado). Aunque no disponemos, por el momento, de resultados derivados de evaluaciones en contextos experimentales $(3,56,70)$, tales empresas parecen asegurar también el acceso y mantenimiento en el empleo de un número significativo de personas con graves problemas de salud mental, en sociedades que, como las europeas, tienen mercados laborales diferenciados del americano (con tasas de desempleo habitualmente superiores y pautas de empleo de mayor estabilidad, en general).

Un resumen de las características generales de este tipo de empresas puede verse en el CUADRO III . Características que adquieren peculiaridades en cada país (17,33,6971) al tener que adaptarse a diferentes contextos y legislaciones laborales, adoptando también diferentes formas jurídicas (cooperativas, por ejemplo, como en el caso de I talia). En concreto, en el nuestro siguen viéndose obligadas a asumir la anacrónica e inadecuada figura de los «centros especiales de empleo». ${ }^{5}$

Hay que señalar también, a pesar de diferencias conceptuales y de contexto, la existencia de distintas experiencias de desarrollo de iniciativas de autoempleo, relacionadas con el movimiento asociativo, tanto en USA como en Canadá $(73,74)$.

Aunque falta un análisis detallado de similitudes y diferencias, así como comparaciones experimentales de sus resultados, ambos modelos constituyen en nuestra opinión las perspectivas actualmente más prometedoras, en las que, por otra parte, no resulta difícil encontrar principios comunes de utilidad para nuestro trabajo: empleos reales con responsabilidad y retribución reales, empresas competitivas, trabajadores con y sin enfermedad mental y apoyos individualizados y sostenidos en el tiempo, basados especialmente en la interacción cotidiana y la formación en el propio puesto de trabajo.

\section{Factores predictivos y repercusiones}

A pesar del esfuerzo realizado y tras muchos años de investigaciones sobre el tema, la búsqueda de variables «predictoras» de éxito o fracaso de los programas de empleo ha dado pocos resultados concretos. De hecho como consecuencia, probablemente inevitable, de la heterogeneidad de poblaciones, programas y metodologías de evaluación $(75,76)$, muchos de los resultados publicados parecen en no pocas ocasiones contradictorios, por lo que resultan a menudo difíciles de tener en cuenta en la formulación de políticas y programas de intervención. Sin embargo, algunos de ellos sí pueden resultar de utilidad: 
a) Durante mucho tiempo se ha hecho hincapié en la escasa importancia del diagnóstico y/ o la sintomatología a la hora de predecir las posibilidades de obtener empleo, como resultado de un programa de rehabilitación y/ o integración laboral $(43,49)$. Sin embargo, desde hace unos años, empieza a haber información de relevancia creciente sobre las mayores dificultades de personas con esquizofrenia, comparadas con las que presentan otros tipos de problemas mentales $(35,77$ 79), y de modo especial, dentro de ese grupo diagnóstico, el de aquellas que presentan un claro predominio de sintomatología negativa y escasas habilidades sociales $(78,80,81)$, aunque no hay concordancia absoluta entre los distintos estudios (82). Pero es, sobre todo, la asociación de malos resultados en términos de empleo con distintos tipos de alteraciones cognitivas, la que parece mostrar mayor consistencia en los estudios más recientes $(78,80,83-87)$, con implicaciones importantes en la evaluación funcional y el desarrollo de intervenciones rehabilitadoras y de apoyo al empleo $(80,88)$.

b) Sigue sin haber datos concluyentes sobre la asociación del éxito o fracaso de los programas de empleo con la mayoría de las variables sociodemográficas habituales $(79,80,82)$, aunque parece confirmarse en distintos estudios el papel de la historia laboral previa a la hora de predecir el probable éxito de aquellos $(43,80)$. Sin embargo, al igual que sucede con el menor número y duración de episodios de hospitalización (43), no está claro si se trata de factores causales o meros indicadores de una posible «baja afectación», cuyas causas siguen siendo bastante poco conocidas.

c) Parece mantenerse también la dificultad para obtener información útil para los programas de empleo basada en el funcionamiento en otras áreas funcionales $(18,89)$ y, especialmente, en otros contextos como el hospitalario (43) 0 , por extensión, otros servicios sanitarios. Sí parece, en cambio, tener algún valor (aunque con peso variable) la adquisición y mantenimiento de algunas habilidades de ajuste laboral, antes del inicio de los programas (77).

d) Y, finalmente, como conclusión que parecería obvia pero no siempre tenida en cuenta, diversos estudios resaltan la importancia básica del «deseo de trabajar», de que el empleo sea un objetivo real para la persona concreta que entra en el programa $(35,43,53,90)$. Podríamos estar aquí ante un factor clave que hace perder peso al efecto de muchos de los restantes factores, cuya contribución sólo parece ser constatable una vez controlado éste (79). Lo que obligaría a prestar más atención a las dimensiones más subjetivas relacionadas con el empleo, tanto en la investigación $(43,89)$ como en la intervención $(53)$. Hay bastante coincidencia en distintos estudios en destacar el elevado nivel de realismo en las expectativas de las personas con enfermedad mental grave interesadas por el empleo $(20,27,79,90)$, así como en relacionar el ajuste laboral y el mantenimiento en el empleo (dimensión diferente de la de su obtención, pero con factores no 
muy distintos $(62,67)$ con dos aspectos que tienen en común un importante componente subjetivo, como es el grado de satisfacción: la adecuación a experiencias previas y preferencias del paciente (91-93) y algunos factores estructurales no siempre bien definidos que tienen que ver también con la interacción social en el espacio de trabajo $(94,95)$.

La conclusión general, en la que coinciden la mayoría de los expertos, es la de la inexistencia de criterios de exclusión, considerando una política de no-exclusión a priori ( «zero-exclusion policy») como uno de los componentes clave para el éxito de los programas de empleo con apoyo (59).

Para finalizar este repaso, hay también evidencia acumulada con respecto a la repercusión del empleo en determinados aspectos clínicos y de funcionamiento social, de las personas con trastornos de tipo psicótico (16). Así, parece razonable afirmar que, aunque no estén claros los efectos directos sobre la sintomatología positiva (ni mejorías ni descompensaciones) y negativa (parece haber en ocasiones ligeras mejorías), sí que se constatan repercusiones positivas en áreas como el funcionamiento social, el grado de satisfacción, la autonomía personal, la autoestima y, en definitiva, la calidad de vida $(20,29,30,92,93,96)$.

\section{Algunas consecuencias prácticas}

Intentando sintetizar todavía más el conjunto de información que acabamos de revisar, para poder extraer de ella algunas consecuencias operativas que pudieran resultarnos útiles, a pesar de las inevitables diferencias contextuales, consideramos razonable hacer hincapié al menos en los siguientes aspectos:

a) El colectivo de personas con trastorno mental severo y, especialmente su componente más numeroso y problemático constituido por las personas con diagnóstico de esquizofrenia, parece caracterizarse, en general y muy especialmente en el terreno del acceso a la actividad productiva y el empleo, por una considerable complejidad y variabilidad sincrónica y diacrónica (39-42). Complejidad y variabilidad que afectan a características, dificultades y factores implicados, contribuyendo a conformar, entre otras cosas, un grupo de personas muy diferente de otros colectivos con dificultades de empleo.

b) Hacer frente a esa situación exige procesos igualmente complejos e individualizados que permitan intervenciones múltiples, dependientes de diversas agencias (sanitarias y no sanitarias), algunas de las cuales deben ser sostenidas en el tiempo y, en conjunto, bien articuladas entre sí, tanto en el diseño y desarrollo general cuanto en su aplicación a personas concretas $(3,39,56,66,95,97)$. Procesos cuya efectividad cuenta con una creciente base experimental y para los que no parecen existir, a priori, factores de exclusión, por muy difíciles y prolongadas que parezcan las dificultades de partida de algunas personas (59). 
c) Aunque muchos aspectos distan de estar suficientemente contrastados, hay ya un acúmulo considerable de conocimientos útiles para el desarrollo de tales programas. Entre todos ellos merece la pena hacer mención al menos de los siguientes: la importancia de tener en cuenta las experiencias, deseos y preferencias del usuario $(35,43,53,90)$, la importancia de la motivación (90-95) individual y colectiva (que puede funcionar como refuerzo a la individual) a todo lo largo del proceso, la necesidad de fijar tan pronto como se pueda objetivos claros en torno a empleos concretos (53-55,65-67), la mayor utilidad de realizar la formación en el contexto real del empleo $(53-55,68)$, la necesidad de apoyos flexibles, individualmente adaptados y muy prolongados en el tiempo (60), y la conveniencia de partir de los contextos sociales y económicos concretos, tanto para el desarrollo de los programas como para la elección de actividades productivas y sectores de empleo cuando se pretende poner en marcha empresas sociales (33,69-71).

d) Dado el papel múltiple que puede jugar el trabajo en la vida de estas personas y aunque el ideal sigue siendo una actividad coherente, retribuida y socialmente valorada, parece necesario disponer de alternativas graduadas que permitan una mejor adaptación a la variabilidad del colectivo $(3,28,56,57)$. En concreto, aunque sigue habiendo controversias sobre la utilidad de espacios estrictamente ocupacionales, dada su escasa capacidad de facilitar el acceso al empleo real y su no despreciable riesgo de institucionalismo, puede ser útil disponer de ellos en el marco de un programa unificado, integrándolos como un escalón más que puede servir de paso (hacia arriba o hacia abajo), desde y hacia otros niveles (57).

Como venimos repitiendo a lo largo del texto, dichas condiciones exigen procesos complejos y de relativamente larga duración, que no parecen demasiado compatibles con los procedimientos habituales de las llamadas «agencias de empleo». Organizaciones que, por extrapolación de experiencias en otros colectivos, pretenden solucionar el problema sobre la base de distintas combinaciones de una bien intencionada y aparentemente novedosa «ideología de la integración» (con afirmaciones del tipo: «lo específico segrega en lugar de integrar», «hay que enfatizar los aspectos positivos y no los negativos», o «el principal problema es la falta de flexibilidad y adaptación al nuevo contexto laboral y tecnológico») y actividades limitadas, algunas de las cuales pueden ser útiles en un marco más amplio, pero absolutamente insuficientes separadas de éste: evaluación, orientación, formación profesional, búsqueda de empleo, trabajo en prácticas, etc. 
CARACTERÍ STI CAS BASICAS DE UN PROGRAMA OCUPACI ONAL-LABORAL PARA PERSONAS CON TRASTORNO MENTAL SEVERO. ASPECTOS ESTRUCTURALES

Sobre la base de la información que acabamos de resumir y a partir de nuestra experiencia en Andalucía $(4,17,98)$, con utilización en ocasiones textual de parte del material escrito utilizado en ella (4), pasamos a exponer nuestras propuestas con respecto a algunos de los elementos básicos que, en nuestra opinión, deberían servir para la elaboración y desarrollo de un programa ocupacional-laboral para personas con trastorno mental severo.

J ustificada en las paginas anteriores la necesidad de tal programa, considerado globalmente, parece que su objetivo general debería ser incrementar la cantidad y calidad de actividad productiva y empleo de dichas personas, mediante distintas actividades orientadas a conseguir el mantenimiento en el empleo de aquellos que lo tienen, el acceso al mismo de los que no lo tienen pero pueden llegar a tenerlo y, secundariamente, el desarrollo de actividad ocupacional para los que no pueden conseguirlo o mantenerse en él.

Sobre esta base y teniendo en cuenta que las características concretas del mismo pueden variar de manera significativa según distintos factores contextuales, hay sin embargo algunos elementos comunes sobre los que vamos a insistir a continuación.

1. Destinatarios del programa

El establecimiento de los perfiles cuantitativos del programa, lo que podemos llamar «cuantificación de necesidades», es difícil de hacer de manera rigurosa, entre otras cosas por ser, probablemente, muy variable en función del contexto socioeconómico general y de servicios $(4,25)$ en el que pretendamos movernos. En cualquier caso, hay que tener en cuenta, dentro de la prevalencia de personas con trastornos psicóticos, la existencia de tres subpoblaciones que presentan problemáticas específicas y requieren por tanto estrategias relativamente diferenciadas:

a) La de los pacientes con empleo (habitualmente no más de un 10-15\% del total), en los que se trata de intentar impedir que lo pierdan (3) mediante un trabajo a varias bandas (paciente, familia, profesionales sanitarios y entorno laboral).

b) La de los pacientes que tuvieron empleo pero lo han perdido a consecuencia de su enfermedad (cifra mucho más variable según el contexto socioeconómico, pero relativamente más alta de lo que en principio pudiera pensarse (99), población en principio más favorable para volver a trabajar.

c) Y la de los pacientes que nunca tuvieron un empleo, población teóricamente con mas dificultades pero que no por ello debe excluirse a priori del programa (59).

\section{Intervenciones necesarias}

Si tenemos en cuenta todo lo que hemos dicho anteriormente, para intentar cubrir las necesidades de actividad productiva y empleo de las distintas personas que 
componen dichos colectivos, deberíamos estar en condiciones de ofrecerles un amplio abanico de intervenciones, diferenciadas pero adecuadamente integradas en programas individualizados $(3,56)$.

Como hemos planteado ya en otras ocasiones $(4,98)$, consideramos que una propuesta razonable debería incluir básicamente los siguientes tipos de intervenciones, que, dependiendo de los usuarios y de los contextos concretos, pueden realizarse ya sea en estructuras de tipo general, ya en estructuras específicas para personas con trastorno mental severo:

1. Un adecuado Tratamiento sanitario, con una orientación general que, más allá del control sintomatológico, busque favorecer, en la mayor medida posible, la permanencia en la comunidad.

2. Los programas específicos de Rehabilitación Psicosocial, sobre los que, aunque deberían estar incluidos en el apartado anterior $(11,12)$, queremos insistir tanto por su importancia intrínseca y habitual «olvido», como por servir de base para las intervenciones más específicas en el área laboral.

3. La Evaluación de necesidades y posibilidades del usuario en el ámbito de la ocupación y el empleo, incluyendo historia laboral previa, situación actual, aspiraciones y deseos, etc., así como la elaboración de un itinerario personalizado.

4. Distintas actividades dirigidas a la Orientación vocacional de cada usuario, ayudándole a definir sus posibilidades en el contexto concreto en que se encuentra.

5. Los programas de la Adquisición de hábitos laborales básicos, en el mínimo indispensable para su posterior integración en actividades laborales.

6. La posibilidad de desarrollar Actividad ocupacional, ya sea como escalón previo, como actividad de transición en situaciones de retroceso en el proceso de integración, 0 , en no pocos casos, como actividad prácticamente permanente.

7. Distintas actividades y programas de Formación básica y compensatoria, para superar dificultades y lagunas concretas en el proceso de aprendizaje previo de algunos usuarios.

8. Los programas específicos de Formación profesional que correspondan en cada caso, de acuerdo al itinerario formativo definido para cada usuario.

9. La posibilidad de desarrollar distintas formas de Trabajo supervisado, de carácter temporal, como fase de transición entre el periodo formativo y el de empleo.

10. Distintas actividades de Orientación y apoyo en la búsqueda de empleo, incluyendo el desarrollo de las habilidades necesarias para ello.

11. La disponibilidad de una panoplia, lo más amplia y variada posible, de Alternativas de empleo, desde modalidades con distinto nivel de protección hasta al empleo ordinario en sus diversas formas (incluyendo el autoempleo).

12. Y, finalmente, distintos mecanismos de Apoyo en el puesto de trabajo, con intervenciones potenciales no solo sobre el paciente si no también, y según los casos, sobre el entorno familiar, profesional y laboral. 


\section{Dispositivos y niveles organizativos convenientes}

Para el desarrollo de dichas prestaciones hay que contar con un número variable de «dispositivos», entendiendo por tales estructuras concretas, en las que se desarrollan funciones específicas con recursos materiales y humanos más o menos «tipificables», aunque en algunos casos (por ejemplo una Empresa Social) la utilización del término «dispositivo» no resulte del todo adecuada.

Basándonos una vez más en nuestra experiencia en Andalucía $(4,98)$, aunque sin perder de vista la inevitable variabilidad de los mismos, en función de los diversos contextos administrativos, el CUADRO IV ofrece una visión esquemática ordenándola a efectos didácticos en cinco niveles, uno de los cuales corresponde directamente a los servicios de salud mental.

Así, el esquema contempla básicamente los siguientes «niveles» y «dispositivos» 0 estructuras organizativas, si bien volvemos a insistir en que, evidentemente, son perfectamente posibles otras combinaciones, tipologías y denominaciones concretas:

a) Un nivel sanitario, al que, además de la atención global necesaria en cada caso, incluyendo el seguimiento individualizado a lo largo del proceso de integración laboral, le corresponden algunas funciones que podemos considerar formalmente «previas» al mismo, como son el tratamiento comunitario, la rehabilitación psicosocial y una primera orientación vocacional. Funciones que en nuestro modelo corresponden básicamente a los Equipos de Salud Mental y las Unidades de Rehabilitación, sobre cuya dependencia sanitaria hemos insistido en otras ocasiones $(11,12)$

b) Un nivel ocupacional - prelaboral, al que, sin perjuicio de que algunos de los dispositivos que incluimos en él, puedan tener otras funciones en el proceso de atención comunitaria de sus usuarios, cumple también algunas específicas en el proceso de integración laboral, como son las de evaluación y orientación, adquisición de hábitos básicos y la actividad ocupacional (transitoria y/o alternativa). Para ello son precisos algunos dispositivos específicos del tipo de los Talleres yl - Centros ocupacionales, si bien hay que estar atentos a controlar sus riesgos de institucionalismo y desmotivación. Para intentar contrarrestarlos parecen especialmente útiles algunos mecanismos, como son la revisión periódica de su orientación y funcionamiento, la búsqueda de la mayor orientación a la producción y el mercado posibles en cada caso, así como la implicación activa de los usuarios en su desarrollo y dinámica cotidiana $(50,57,61)$.

c) Un nivel formativo, cuya función, sobre la base de un itinerario individualizado y haciendo uso tanto de recursos específicos como «normalizados», es la de completar la formación de los usuarios a través de actividades de formación básica y compensatoria, formación profesional, trabajo supervisado 0 «en prácticas» y orientación en la búsqueda de empleo. Actividades que pueden desarrollarse a través de Cursos de educación general y compensatoria, Cursos de Formación profesional, y distintos tipos de Proyectos formativos y «formativo-empresariales». ${ }^{6}$ 
d) Un nivel laboral, en el que se trata de facilitar oportunidades graduadas de empleo, en sentido estricto, incluyendo empleo de transición y en entornos protegidos, empleo apoyado y empleo ordinario. Para ello se consideran, como «dispositivos» u organizaciones específicas, Empresas de inserción, Empresas sociales y Equipos de Apoyo al Empleo

e) $Y$, finalmente, un nivel de coordinación y apoyo, en el que tienen cabida al menos dos tipos de dispositivos:

- los Servicios de Orientación y Apoyo al Empleo, que deben permitir la coordinación y supervisión del conjunto del programa en niveles territoriales operativos (provincia, área de salud, etc.); además de actuar como «filtro» en los flujos entre sus diversos niveles, y de facilitar algunas prestaciones específicas, como son la evaluación y orientación vocacional, el diseño de itinerarios personalizados y la coordinación con el nivel sanitario ${ }^{7}, y$,

- alguna estructura específica de apoyo a las empresas sociales, ${ }^{8}$ facilitando servicios de orientación, asesoría y apoyo a la gestión de las mismas.

\section{Profesionales necesarios}

Cuanto más compleja sea la red que integra el programa, mayor será la diversidad de perfiles profesionales implicados, la mayor parte de los cuales se diferencian claramente de los sanitarios tradicionales y requieren, además, algún tipo de entrenamiento específico para su relación con personas con trastornos mentales severos (también aquí, como en otros programas de apoyo social, los modelos basados en la psicoeducación familiar pueden ser bastante útiles). Sin olvidar que algunas actividades pueden recaer durante bastante tiempo en personal polivalente, y que distintos tipos de profesionales actuales pueden cumplir diferentes funciones de las establecidas en el programa, consideramos necesario mencionar la necesidad, al menos, de:

a) Monitores de Talleres y Centros ocupacionales, que exigen un apoyo y orientación específicos para contrarrestar las inevitables tendencias al institucionalismo.

b) Monitores de formación profesional, básicamente expertos en la actividad de que se trate, con sensibilidad hacia el colectivo y habilidades didácticas.

c) Técnicos en rehabilitación vocacional, fundamentalmente Terapeutas Ocupacionales y Psicólogos.

d) Orientadores laborales, expertos en los procesos que se dan en el mercedo laboral.

e) Gerentes o Directores de Empresas sociales, básicamente «empresarios», pero también con sensibilidad hacia este colectivo.

f) Trabajadores de empresas sociales, que son habitualmente trabajadores del sector que corresponda, pero que asumen funciones de tutela y apoyo que pueden requerir algún tipo de entrenamiento y soporte.

g) Y distintos tipos de Monitores de apoyo, especialmente en los programas de empleo en el mercado laboral ordinario. 


\section{Criterios de inclusión en el Programa}

Aún defendiendo una política general de «no-exclusión» a priori para la entrada en el programa, parece necesario establecer algunos criterios que permitan regular el acceso a sus distintas actividades, con la suficiente flexibilidad para no dificultar más aún las ya de por sí difíciles situaciones de muchos usuarios. Así, partiendo de la habitual limitación de recursos existentes en nuestros contextos cotidianos, parece lógico tener en cuenta aspectos como:

a) La gravedad del problema de salud mental en general y sus repercusiones sobre la actividad y el empleo, de cara establecer prioridades de intervención desde un programa especializado.

b) La relación del futuro usuario con los servicios públicos de salud mental, a los que debe corresponder su evaluación inicial, la indicación técnica y la responsabilización del seguimiento sanitario (plan individualizado con componentes rehabilitadores)

c) Y la capacidad para seguir las actuaciones específicas del programa y/o subprogramas que se determinen como más convenientes, lo que significa al menos un mínimo de motivación, la ausencia, o posibilidad de control, de manifestaciones sintomatológicas que dificulten la relación con el personal y/o los compañeros, y una razonable expectativa de desempeño de las actividades incluidas.

Es evidente que los distintos componentes que configuran este último "criterio» van a depender de manera muy marcada del nivel concreto del programa que se contemple en cada caso, siendo más complejos a medida que nos acercamos a los más estrictamente laborales. Así, por ejemplo, la participación en algunas actividades de un taller ocupacional no exige demasiados requisitos en términos de motivación o capacidad, ya que esos son aspectos trabajables a este nivel, como prolongación de su programa de rehabilitación. Sin embargo, el aprovechamiento de un curso estructurado $y$, en mayor medida, el acceso al empleo en un medio específico 0 general, van a exigir una motivación clara y un conjunto de capacidades mínimas mucho mayores, si queremos que el aprendizaje y la integración sean reales.

Por otro lado, la ausencia de manifestaciones sintomatológicas distorsionantes debe verse con carácter relativo, en la medida en que intenta eliminar interferencias en el proceso de aprendizaje o desempeño de la tarea por parte del propio usuario, a la vez que funciona como mecanismo protector de los derechos de los demás participantes. En cualquier caso tiene un carácter estrictamente temporal, implicando, lógicamente, un plan individualizado coordinado con el servicio sanitario para su control.

En resumen, aunque podríamos decir que prácticamente todas las personas con trastorno mental severo serían teóricamente susceptibles de incorporarse a un programa ocupacional-laboral, ello no significa que puedan hacerlo en cualquier mo- 
mento ni en cualquiera de sus niveles, dispositivos o subprogramas. Precisamente la concepción aquí presentada, que contempla una red de servicios y actuaciones con distintos niveles e itinerarios individuales, pretende servir de base para las distintas evoluciones deseables y posibles de los usuarios. Lo que debe permitir contrapesar dos riesgos simétricos: el rechazo, por un lado, de las personas que, por tener más dificultades, más necesitan este tipo de intervenciones y apoyos; y, por otro, la mala utilización de recursos que significa situar en algunos programas a personas que no pueden beneficiarse de ellos, privando de esa posibilidad a quienes si podrían hacerlo.

Todo ello sin olvidar que, como se ha señalado anteriormente, en la mayor parte de estos aspectos no disponemos todavía de criterios objetivos que permitan predecir de manera rigurosa las evoluciones individuales, lo que obliga a ser cautos y flexibles en la toma de decisiones sobre entradas, tiempos de permanencia, cambios internos y salidas del Programa y de cada uno de sus dispositivos.

\section{Funcionamiento e integración del programa}

A este respecto, hay que tener en cuenta que lo que se trata de conseguir es que, tanto el conjunto del programa como sus distintos dispositivos y actuaciones, se atengan a los objetivos generales de aquel, en la concreción correspondiente a cada nivel y usuario individual. Es decir, que, en la mayor medida posible:

a) Cada usuario cuente con un itinerario individualizado que, en función de sus intereses y capacidades personales y de las posibilidades del medio, le permita utilizar los recursos disponibles para alcanzar el nivel más adecuado posible de integración a corto, medio y largo plazo.

b) Cada dispositivo y/o subprograma consiga un nivel razonable de funcionamiento, maximizando su capacidad de dar respuesta a las necesidades de sus usuarios, lo que significa siempre algún grado de compromiso en cuanto al número, homogeneidad-heterogeneidad de aquellos y especificidad-polivalencia de las actuaciones.

c) Y, en cada ámbito territorial, el conjunto de recursos funcione de manera integrada, cubriendo de la mejor manera posible las distintas necesidades del colectivo.

Para ello, además de los mecanismos de formación continuada y apoyo a los profesionales y de la relación con los servicios de salud mental (a la que haremos referencia a continuación), es esencial que haya una coordinación estricta entre los distintos componentes del programa en los ámbitos territoriales pertinentes. A este respecto, parece que los anteriormente mencionados Servicios de Orientación y Apoyo al Empleo pueden ser una respuesta razonable a esta necesidad de coordinación, interviniendo tanto como supervisión y apoyo al funcionamiento de cada dispositivo y sus distintas relaciones internas y externas, como en el seguimiento individualizado de las personas que utilizan el programa. 


\section{Coordinación externa}

Un aspecto clave es la coordinación con los servicios de salud mental ya que no podemos olvidar que este programa, en tanto que dirigido a facilitar la integración social de personas con trastorno mental severo, tiene sentido y posibilidades de éxito únicamente en el marco de un conjunto más amplio de políticas e intervenciones dirigidas a la atención comunitaria a estas personas $(11,12)$.

Ello exige una vinculación estricta con los servicios de salud mental (25), vinculación que afecta a los distintos momentos en que se articula el programa, incluyendo tanto los aspectos generales de planificación y evaluación periódicas de recursos y actuaciones, cuanto las intervenciones individuales sobre los distintos usuarios, en cada uno de los dispositivos y actividades concretas. Lo que exige también, una estructura organizativa clara que facilite la coordinación, incluyendo los distintos niveles territoriales de articulación del programa, desde el más general al de cada dispositivo y usuario.

En nuestra manera de entender esta relación $(4,7,25)$ y en lo que respecta a los distintos pasos que requieren coordinación en el seguimiento individualizado de los usuarios, hay que tener en cuenta que:

a) Deben ser los servicios de salud mental, habitualmente a través de sus Equipos comunitarios y Unidades de Rehabilitación, los que deben establecer la indicación de entrada en el programa, sobre la base de una evaluación inicial y en el marco de una estrategia individualizada de rehabilitación.

b) Son también ellos los que, con las modalidades y mecanismos concretos que se definan en cada caso, deben asegurar la atención sanitaria individual que corresponda.

c) El itinerario individual previsto para cada usuario debe elaborarse contrastando esa primera indicación con la evaluación del Servicio de Orientación y Apoyo al Empleo, que permite, además, la consideración de las posibilidades concretas que ofrecen los recursos disponibles.

d) Y, a lo largo de todo el proceso (que puede incluir el paso por distintos recursos y el escalonamiento de muy diferentes intervenciones) pueden ser necesario reevaluar en más de una ocasión la situación y modificar el itinerario previsto, lo que obliga de nuevo a coordinarse para la toma de decisiones y el seguimiento de las intervenciones establecidas.

Del mismo modo, en la medida en que las actividades de integración laboral de personas con dificultades están experimentando un importante desarrollo en estos momentos, el programa debe mantener una adecuada interacción con todo ese campo, sin renunciar a contemplar la especificidad de las actuaciones necesarias para el colectivo con el que nos movemos, pero integrándose en un contexto más general.

Así, sin pretender ser exhaustivos, hay que tener en cuenta la necesidad de coordinación con las distintas estructuras administrativas con competencias sobre el 
empleo (autonómicas y estatales), con organismos donde se definen $y / 0$ articulan programas de apoyo al empleo para distintos colectivos (organismos representativos tipo CERMI, organizaciones como ONCE, asociaciones de Centros Especiales de Empleo, etc.), así como con distintas organizaciones sociales implicadas (Sindicatos y organizaciones empresariales).

Todo ello sin olvidar las necesidades de coordinación con otros programas de apoyo social de dependencia no sanitaria, en temas como la residencia, ocio, asociacionismo, etc.

\section{Seguimiento y evaluación de resultados ${ }^{9}$}

La importancia de la evaluación es cada vez más evidente en distintos campos de intervención, especialmente cuando se trata de servicios que consumen recursos públicos, habitualmente escasos y susceptibles, por tanto, de usos alternativos que deben justificarse razonablemente (104). De manera especial, y como hemos hecho referencia en otras ocasiones $(11,12)$, la atención comunitaria en salud mental se beneficiaría de una aproximación tecnológica que supere el exceso de artesanado, rutina y endeble fundamentación científica de no pocas de sus prácticas profesionales. En ese sentido, la necesidad de evaluación no deriva ni única ni principalmente del interés de los «administradores» y «políticos», responsables ante los ciudadanos a la hora de pedir cuentas de nuestro trabajo, sino que debería ser una exigencia cotidiana del conjunto de los profesionales (105). De hecho, la evaluación de nuestras intervenciones no debe verse sólo como una exigencia «externa» sobre el uso de recursos públicos, si no que, además de que tal preocupación debe ser incorporada por todos nosotros, tenemos que considerarla como un insustituible mecanismo de aprendizaje y autocorreción que debe formar parte indisociable de todas y cada unas de nuestras actuaciones $(106,107)$.

Sin embargo, la situación habitual está bastante alejada de estos planteamientos. Tanto en el campo de la atención en salud mental como, específicamente, el de los programas de empleo para personas con trastorno mental severo, no abundan las evaluaciones rigurosas de intervenciones, dispositivos y programas. Situación que se agrava en nuestro país, donde a las dificultades metodológicas generales se une un notable retraso en este y en otros campos de intervención sanitaria y social (108). ${ }^{10}$

Las dificultades reales no deben ser obviadas. Tanto la aplicación de la metodología general de evaluación de intervenciones y programas al campo de la salud mental $(103,105,108)$, como el desarrollo específico de metodología en el campo del empleo, presentan problemas aún no resueltos que dificultan la fundamentación racional de no pocas intervenciones. A pesar de ellas, sin embargo, el desarrollo de la investigación relacionada con los programas de empleo de personas con distintos tipos de enfermedades y trastornos mentales graves (algunos de cuyos resultados hemos resumido en la primera parte del capítulo) ha ido creciendo en los últimos 
años, sin que, sin embargo, nuestro país se haya incorporado como corresponde a esta tendencia. Lo que produce, entre otras cosas, una carencia mayor de metodología e instrumentos concretos adaptados a nuestro contexto.

Sin pretender desarrollar el tema, que exigiría al menos un artículo completo para especificar algunos de sus aspectos más relevantes, vamos a intentar solamente hacer una breve referencia a los elementos que creemos necesario tener en cuenta para enriquecer nuestro trabajo futuro.

En primer lugar parece que es preciso un esfuerzo por «desacralizar» el tema. Aunque la evaluación es un campo de desarrollo con aspectos complejos y metodológicamente sofisticados, también tiene dimensiones cotidianas compatibles con la práctica habitual de los profesionales, si se hace un pequeño esfuerzo de sensibilización y aprendizaje metodológico (103). En realidad, incluso incorporando aspectos de investigación (en definitiva, intentar dar respuesta de manera sistemática a preguntas de interés para nuestra práctica), bastantes aspectos de la evaluación de lo que hacemos deben formar parte de nuestras intervenciones y pueden cubrirse con un cierto rigor, pero no necesariamente con procedimientos excesivamente complejos (107).

En segundo lugar, habría que recordar los elementos de metodología general $(104,105,108-113)$ que pueden sernos útiles, adaptándolos a nuestro peculiar campo de trabajo. Así parece necesario hacer referencia a algunos aspectos para evitar confusiones más frecuentes de lo deseable:

a) la diferencia entre «niveles» 0 «dimensiones» de la evaluación, con referencia obligada a la ya clásica formulación de estructura, proceso, resultados (110). En relación con esto, es necesario diferenciar tres aspectos:

- el seguimiento o monitorización de las actividades relevantes del programa, mediante un sistema de información que las traduzca en indicadores,

- la definición de estándares de calidad en una serie de dimensiones o criterios

clave para establecer la estructura y funcionamiento deseables en distintos dispositivos e intervenciones, $y$,

- la evaluación de resultados propiamente dicha.

Siendo, sin embargo, este último el que puede permitir dar respuestas razonables al segundo (estableciendo estándares de calidad en función de que dan lugar a resultados útiles) y orientar el desarrollo del primero (identificando actividades e indicadores relevantes, precisamente por su contribución a obtener dichos resultados);

b) los diferentes aspectos que cabe incluir también dentro de la evaluación de resultados, según intentemos medir por ejemplo eficacia, efectividad, eficiencia, equidad, satisfacción, etc.;

c) los diferentes diseños metodológicos que ofrece la Epidemiología, según se trate de estudios descriptivos u observacionales, analíticos (cohortes, "casos y con- 
troles») o experimentales (ensayos controlados, ), con resultados de validez creciente pero condiciones de realización más complejas;

d) Ia aplicabilidad creciente de metodología denominada «cualitativa» que permite aproximaciones razonables a aspectos subjetivos y complejos no siempre fácilmente abordables con metodología cuantitativa, pero que requieren procedimientos más rigurosos que nuestra simple opinión personal;

e) el desarrollo de instrumentos para medir variables relevantes, entre las que se incluyen claramente al gunos grupos: sociodemográficas, clínicas, habilidades personales, funcionamiento social, ajuste y rendimiento laboral, satisfacción.

Por último no habría que perder de vista la necesidad de establecer algunas prioridades en nuestro país, que, en nuestra opinión podrían ser básicamente, además de la consideración de la evaluación como un componente obligatorio de éste y otros programas, las siguientes:

a) La elaboración e implantación de un sistema común de indicadores básicos, que permitan el seguimiento comparativo de los distintos programas, en distintos contextos territoriales incluyendo algunos estándares consensuados.

b) La definición también consensuada de algunos criterios y estándares de calidad de distintos tipos de dispositivos y programas de intervención.

c) El desarrollo de un paquete de instrumentos para medir algunas de las variables más relevantes, a través de la traducción y adaptación de algunos de los internacionalmente disponibles, así como la elaboración y validación de otros nuevos.

d) La construcción de un esquema básico de evaluación del programa en su conjunto así como de sus diversas actividades.

e) Y algún estudio multicéntrico comparativo, que incorpore a ser posible alguna evaluación, por simple que sea, de los resultados de distintos programas en diversos contextos territoriales y de servicios.

\section{A MODO DE CONCLUSIONES GENERALES}

Para terminar y aún a riesgo de resultar repetitivos, pensamos que puede ser útil plantear algunas consideraciones generales, a modo de resumen de los aspectos que nos parecen más importantes para ayudarnos a orientar el trabajo de los que nos movemos en este campo.

1. En primer lugar, parece que en los últimos años las personas con dificultades de empleo relacionadas con presentar graves problemas de salud mental, y muy especialmente, aquellas con diagnóstico de esquizofrenia, han avanzado bastante en el reconocimiento de su derecho a acceder a políticas públicas de apoyo en este área. Sin embargo, distintos factores limitan todavía de modo considerable 
el ejercicio práctico y efectivo de ese derecho, especialmente en nuestro país (aunque con marcadas diferencias entre sus distintas Comunidades Autónomas):

a) La persistencia de importantes barreras, algunas de ellas con traducción legal, relacionadas con el estigma social hacia este tipo de personas y que afectan tanto al derecho de ciudadanía global de las mismas como, específicamente, a su acceso al empleo.

b) El todavía muy insuficiente desarrollo general de servicios públicos de salud mental, de base y orientación comunitaria, tanto sanitarios como de apoyo social y con capacidad real para una atención efectiva, global e individualizada y con un adecuado componente de rehabilitación $(114,115)$.

c) La escasa responsabilidad que habitualmente asumen las distintas administraciones públicas en el desarrollo de programas y servicios de apoyo al empleo para este colectivo; responsabilidad que no puede ser substituida por las iniciativas del sector «no gubernamental» si no cuenta con una red más sólida y general.

d) Y la falta de un modelo de intervención en este campo razonablemente consensuado y apoyado en los conocimientos científico-tecnológicos hoy existentes.

Este último aspecto nos preocupa especialmente ya que, como de manera insistente venimos repitiendo a lo largo del texto, un funcionamiento inadecuado (que no tenga en cuenta las peculiaridades del colectivo) no sólo no resuelve sus problemas, si no que tiene una gran probabilidad de terminar convirtiéndose en una nueva barrera.

2. Es verdad que no tenemos, a la hora de facilitar el acceso y mantenimiento en el empleo de personas con trastornos mentales graves, una tecnología propiamente dicha (conocimientos teóricos, procedimientos prácticos y agentes entrenados) para lograrlo. Pero sí que vamos disponiendo de elementos que nos pueden permitir avanzar un poco más en su desarrollo, a través de la difusión de información nacional e internacional, del análisis riguroso de nuestras experiencias y, sobre todo, de la discusión en común entre todos los que estamos implicados en este tipo de trabajo, desde distintas posiciones y territorios.

3. Como elementos básicos de un modelo común razonable, que nos permitiría avanzar un poco más a todos los interesados en este campo de trabajo, pensamos que hay que incluir los siguientes:

a) La constatación de que el colectivo de potenciales usuarios / beneficiarios de estos programas es profundamente heterogéneo en varias dimensiones, como son la situación laboral en que se encuentran (en riesgo de perder el empleo, habiéndolo perdido y sin haberlo tenido nunca), las dificultades y capacidades concretas que presentan, sus aspiraciones, expectativas y deseos, así como una gran variedad de aspectos contextuales (familia, servicio, situación social, localización geográfica, etc.). 
b) La necesidad por tanto de una gran variedad de programas, intervenciones y agentes implicados. A este respecto, además, las necesidades concretas dividen de nuevo al colectivo entre los que pueden resolver sus problemas por sí mismos o con un «pequeño empujón», los que necesitan intervenciones básicamente compatibles con agencias generales o inespecíficas, los que necesitan intervenciones complejas y especializadas, habitualmente no disponibles en las agencias normales, y, finalmente, los que necesitan alternativas diferentes al empleo. Clasificación poco precisa en términos operativos pero que puede ser útil como orientación general.

c) Lo mismo sucede en relación con los tipos de empleo, donde hay necesidad de combinar empleos en empresas «ordinarias» (sin apoyo o con apoyos puntuales, de los que el empresario y los compañeros no tienen porque enterarse), programas específicos de empleo con apoyo, empleo en empresas sociales, como transición hacia empresas «ordinarias», empleo permanente en empresas sociales y distintas modalidades de itinerarios complejos entre unas y otras, además de trabajos a tiempo parcial, en prácticas, etc.

d) La utilidad (a falta todavía de comprobación experimental de su efectividad y eficiencia) de procesos colectivos de media-larga duración, que combinan de manera programada y en contextos reales de desarrollo productivo y empresarial, actividades de orientación, motivación, formación, trabajo y apoyo. Procesos que requieren, además, una buena coordinación con los servicios de salud mental y para los que algunas experiencias de creación de empresas sociales pueden ser ejemplos de interés.

e) La conveniencia de contar, para ser capaz de abarcar razonablemente esa complejidad de necesidades e intervenciones, con algún tipo de servicio especializado, que trabaje con los casos más complejos y sea capaz a la vez de asesorar y apoyar a los servicios más generales en el manejo de personas que, en la mayoría de los casos, plantean dificultades bastante diferentes de las de otros colectivos.

f) $Y$, por último, la urgencia de desarrollar procedimientos comunes de seguimiento y evaluación de las distintas actividades, dispositivos y programas, que nos permitan avanzar sobre bases científico-tecnológicas en este difícil pero interesante campo de trabajo. 


\section{CUADRO 1. EVOLUCIÓN HISTÓRICA DE LOS MODELOS DE PROGRAMAS DE EMPLEO}

\begin{tabular}{|c|c|c|}
\hline TIPO DE PROGRAMA & CARACTERÍSTICAS & $\begin{array}{c}\text { VENTAJ AS } \\
\text { E INCONVENIENTES }\end{array}$ \\
\hline Laborterapia institucional & $\begin{array}{l}\text { Actividad laboral y pseudolabo- } \\
\text { ral en las instituciones psiquiá- } \\
\text { tricas }\end{array}$ & $\begin{array}{l}\text { - Organización específica } \\
\text { - Desvalorizada e institucionali- } \\
\text { zadora }\end{array}$ \\
\hline Empleo protegido & $\begin{array}{l}\text { Actividad laboral y pseudolabo- } \\
\text { ral en entornos específicos }\end{array}$ & $\begin{array}{l}\text { - Organización específica en } \\
\text { entornos protectores } \\
\text { - Habitualmente no laboral y con } \\
\text { escasos resultados }\end{array}$ \\
\hline Rehabilitación vocacional & $\begin{array}{l}\text { Programas específicos para ac- } \\
\text { ceder a un empleo: } \\
\text { Orientación } \\
\text { Entrenamiento en búsqueda } \\
\text { Formación profesional } \\
\text { Empleos de transición }\end{array}$ & $\begin{array}{l}\text { Buen resultado como entrena- } \\
\text { miento para el acceso al em- } \\
\text { pleo } \\
\text { Escasos resultados para man- } \\
\text { tenerlo sin apoyo prolongado }\end{array}$ \\
\hline Empleo con apoyo & $\begin{array}{l}\text { Empleo inmediato en «empre- } \\
\text { sas normales», con formación } \\
\text { posterior y apoyo externo con- } \\
\text { tinuado }\end{array}$ & $\begin{array}{l}\text { Resultados muy positivos (40- } \\
60 \% \text { ) en experiencias en USA } \\
\text { Dificultades en países con alto } \\
\text { desempleo }\end{array}$ \\
\hline Empresas sociales & $\begin{array}{l}\text { Empleo en empresas específi- } \\
\text { cas, pero que compiten en el } \\
\text { mercado }\end{array}$ & $\begin{array}{l}\text { Ventajas del empleo protegido } \\
\text { y del empleo con apoyo } \\
\text { Buenos resultados en Europa } \\
\text { (Estados del bienestar) pero sin } \\
\text { comprobación experimental }\end{array}$ \\
\hline
\end{tabular}

\section{CUADRO 2. ELEMENTOS BÁSICOS DE LOS MODELOS DE EMPLEO CON APOYO («SUPPORTED EMPLOYEMENT») (53-55)}

Búsqueda rápida de empleo, con formación posterior en el lugar de trabajo

Empleos y retribución normales 0 «de mercado»

- Se tiene en cuenta prioritariamente las preferencias del usuario

- Sistemas flexibles de apoyo, de larga duración

- Estrecha colaboración entre equipo de apoyo y equipo sanitario 


\section{CUADRO 3. CARACTERÍ STI CAS DE UNA EMPRESA SOCIAL (14)}

- Una empresa «normal»:

- Competitiva en el mercado (calidad / coste)

- Auto sostenible

- Con reparto de puestos y retribuciones normales (no-discriminación)

- Pero también una empresa «específica»:

- Clima favorable a la integración activa de personas con dificultades

- Apoyo personal interno y externo (servicios sanitarios)

- Apoyos económicos (Economía social)

- Con forma jurídica variable (Cooperativa, Sociedad Anónima, etc.)

- Y con composición mixta: trabajadores con y sin trastornos mentales

CUADRO 4. NI VELES FUNCI ONALES Y ORGANI ZATI VOS DEL PROGRAMA

\begin{tabular}{|c|c|c|}
\hline NIVELES & DISPOSITIVOS & FUNCI ONES \\
\hline Sanitario & $\begin{array}{l}\text { Equipos / Centros de Salud } \\
\text { Mental } \\
\text { Unidades de Rehabilitación }\end{array}$ & $\begin{array}{l}\text { Tratamiento } \\
\text { Rehabilitación } \\
\text { Orientación general } \\
\text { Seguimiento continuado y apoyo }\end{array}$ \\
\hline Ocupacional - prelaboral & $\begin{array}{l}\text { Talleres / Centros ocupacio- } \\
\text { nales }\end{array}$ & $\begin{array}{l}\text { Hábitos básicos } \\
\text { Actividad no laboral }\end{array}$ \\
\hline Formativo & $\begin{array}{l}\text { Proyectos / Cursos de forma- } \\
\text { ción }\end{array}$ & $\begin{array}{l}\text { Formación básica } \\
\text { Formación profesional }\end{array}$ \\
\hline Laboral & $\begin{array}{l}\text { Empresas Sociales } \\
\text { Equipos de Apoyo al empleo }\end{array}$ & $\begin{array}{l}\text { Empleo de transición } \\
\text { Empleo }\end{array}$ \\
\hline Coordinación general & $\begin{array}{l}\text { Servicios de Orientación y Apo- } \\
\text { yo/ Unidades de Rehabilita- } \\
\text { ción laboral }\end{array}$ & $\begin{array}{l}\text { Evaluación y Orientación } \\
\text { Apoyo } \\
\text { Coordinación del programa }\end{array}$ \\
\hline
\end{tabular}




\section{NOTAS}

1. Con tal de no hablar de «enfermedades» 0 «trastornos mentales» usamos a veces expresiones que rozan el ridículo, como un «enfermo de salud mental» leído hace no mucho tiempo en un informe. Parece que utilizar la expresión «personas con» es un uso adecuado del "lenguaje políticamente correcto» (en el sentido de evitar el refuerzo lingüístico del estigma), así como también decir «Salud mental» en lugar de «Psiquiatría» para referirse al campo de los servicios (enfatizando tanto los cambios en la tipología y orientación de los mismos como su carácter multidisciplinar). Pero también hablar de «trastornos y/ o enfermedades mentales» puede ser perfectamente adecuado y menos «circunloquial» que otras expresiones como «trastornos de salud mental». «Problemas de salud mental» (como concreción del más genérico «problemas de salud») podría ser otra alternativa razonable.

2. Así, por ejemplo, hay bases racionales para sospechar que la incidencia del actual programa Equal del Fondo Social Europeo, en el campo del empleo de personas con trastorno mental severo, va a ser notablemente inferior al que supuso el anterior programa Horizon, tanto en España como en otros países europeos. Y ello a pesar de que las «buenas intenciones» y el sustento ideológico del actual programa (intervenciones integradas en ámbitos territoriales definidos para todos los sectores con dificultades de integración), parecían razonables, frente a las intervenciones específicas para diferentes colectivos que eran habituales en la fase anterior.

3. «Verdaderos empleos con verdaderas retribuciones» (30).

4. Propuesta bastante similar a la que, desde una perspectiva «ecológica» y en el contexto americano, recogen Cook y Razzano en el sentido de generar a modo de «nichos ecológicos» que permitan actividad laboral real en entornos protectores (35).

5. Inadecuada tanto por el efecto estigmatizante de su propia denominación ( «especial»), como por determinadas condiciones legales que impone para tener acceso a las subvenciones previstas (70\% de personas con discapacidad, exigencia que resulta, para nuestro colectivo, imposible en términos de productividad y cuando menos contradictoria en términos de integración). Figura que, por su origen y desarrollo histórico en nuestro país, incorpora además una imagen social negativa, más de empleo protegido que de actividad empresarial real, a pesar de importantes experiencias de autenticas Empresas Sociales que siguen acogiéndose a esta regulación jurídica a falta de otra más adecuada $(17,72)$.

6. Proyectos orientados a la creación de empresas sociales, mediante programas formativos de media duración (17).

7. Funciones que coinciden parcialmente con las de Centros de Rehabilitación Laboral de la Comunidad de Madrid $(100,101)$ 
8. Función que en Andalucía cumple la empresa IDEA, SA (Iniciativas De Empleo Andaluzas), actuando como elemento regulador y supervisor de estrategias específicas para el conjunto de las empresas del grupo $(4,17,102)$.

9. En lo que sigue utilizamos la palabra evaluación para hacer referencia a la «comparación entre lo que se hace y se debería hacer» (103), como traducción habitual del término inglés «evaluation», y no en el sentido de valoración o medida de determinadas dimensiones funcionales, que traduce mejor el término inglés «assessment». Sentidos que tienden a confundirse con cierta frecuencia, especialmente al hablar de rehabilitación, entre otras cosas porque la evaluación de resultados exige medidas precisas de determinadas variables funcionales y clínicas, antes y después de las intervenciones.

10. De hecho son escasas las investigaciones realizadas en este campo, aunque es esperanzador que empiecen a publicarse algunas $(24,75,99)$. Por nuestra parte estamos desarrollando en la actualidad un proyecto financiado por el FIS («Evaluación de los programas de integración laboral para personas con trastorno mental severo en Andalucía») que esperamos nos permita avanzar en la evaluación de distintos componentes del programa, incluyendo el desarrollo, traducción y validación de un conjunto de instrumentos de previsible utilidad para el futuro. Proyecto del que forma parte la revisión bibliográfica resumida en la primera parte de este artículo.

\section{BIBLIOGRAFÍA}

1. Huxley, P.- «Work and mental health: an introduction to the special section». J ournal of Mental Health, 2001,10,4:367-372.

2. Crowther, R. E.,, Marshall, M. y ots. - "Helping people with severe mental illness to obtain work: systematic review». British Medical J ournal, 2001,322: 204-208.

3. Boardman, J; Grove, B y ots.- «Work and employment for people with psychiatric disabilities». British J ournal of Psychiatry, 2003,182:467-468.

4. Fundación Andaluza para la Integración Social del Enfermo Mental.- Criterios para el desarrollo del Programa Ocupacional-laboral. Sevilla, FAISEM, 2000.

5. Toro, A.- «Políticas de empleo para personas con discapacidad». En Lara, L. Y López, M (Ed).- Integración laboral de personas con trastorno mental severo. Sevilla, FAISEM, 2003 (Edición policopiada): 65-69.

6. Lara, L y López, M.- «La provisión de recursos de apoyo social en Andalucía». En García, J, Espino, A y Lara, L - La Psiquiatría en la España de fin de siglo. Madrid, Díaz de Santos, 1998: 213-224.

7. López, M. y Lara, L.(1997).- «La coordinación intersectorial en la atención a la salud mental. Nuevas instituciones y formas de gestión». En Leal, J.- Equipos e instituciones en salud (mental). Salud (mental) de equipos e instituciones. Madrid, AEN, 1997: 149-168. 
8. Corrigan, P. W. y Penn, D. - «Disease and Discrimination: two Paradigms that describe severe mental illness». J ournal of Mental Health, 1997,6, 4:355- 366.

9. Huxley, Py Thornicroft, G.- «Social inclusion, social quality and mental illness». British J ournal of Psychiatry, 2003,182:289-298.

10. López, M.- «Bases para una nueva política en Salud Mental». En García, Espino y Lara, Op. Cit.,1998: 29-39.

11. López, M - «Rehabilitación y red asistencial. Sistemas de atención a la cronicidad en salud mental». En Verdugo, M, López, D y ots.- Rehabilitación en Salud mental. Situación y perspectivas. Salamanca, Amarú, 2002:75-105.

12. López, M; Lara, L y Laviana, M.- «La formación básica en rehabilitación y apoyo social para la atención comunitaria en salud mental». En Espino, A. y Olabarría, B.- La formación de los profesionales de la Salud Mental en España. Madrid, AEN, 2003:269-301.

13. Saraceno, B. - La fine dell'I ntrattenimento. Milán, Etas Libri, 1995.

14. Barker, L. T.- «Community-based models of employment services for people with psychiatric disabilities». Psychosocial Rehabilitation J ournal, 1994,17, 3: 55-65.

15. Penn, D. y Nowlin-Drummond, A.- «Politically correct labels and Schizophrenia: A rose by any other name?». Schizophrenia Bulletin, 2001,27,2: 197-204.

16. Lehman, A. F.- «Vocational Rehabilitation in schizophrenia». Schizophrenia Bulletin, 1995, 21,4: 645-656.

17. López, M.,, Alvarez, F. y Laviana, M.- «Empresas sociales y rehabilitación psiquiátrica en Andalucía». Revista de la Asociación Española de Neuropsiquiatría, 1998, XVIII, 68: 735-747.

18. Mueser, K T, Salyers, M P y Mueser, P R - «A prospective analysis of work in Schizophrenia». Schizophrenia Bulletin, 2001, 27, 2: 282-296.

19. Perkins, R y Rinaldi, M.- « Unemployment rates among patients with long-term mental health problems. A decade of rising unemployment». Psychiatric Bulletin, 2002,26: 295-298.

20. Resnick, SG y Bond, G. «The Indiana J ob Satisfaction Scale: job satisfaction in vocational rehabilitation for people with severe mental illness». Psychiatric Rehabilitation J ournal, 2001, 25,1:12-19.

21. Penn, D. y Mueser, K.- «Research update on the psychosocial treatment of schizophrenia». Am. J. Psychiatry, 1996,153: 607-617.

22. Ruggeri, M., Leese, M. y ots. - «Definition and prevalence of severe and persistent mental illness». British J ournal of Psychiatry, 2000, 177, 149-155.

23. Shepherd, G. - «Avances recientes en Rehabilitación Psiquiátrica». En (40) Aldaz y Vázquez, 1996: 1-22.

24. García, J., Peñuelas, E. y ots.- «La formación y el empleo. Instrumentos básicos para la integración de las personas con trastornos psicóticos». Revista de la Asociación Española de Neuropsiquiatría., 2000, XX, 73: 9-22. 
25. López, M. y Laviana, M.- «Los servicios de salud mental como apoyo a la integración laboral: condiciones funcionales y organizativas». En García y ots, 1998, Op. Cit.: 253-260.

26. Mueser, K T y Tarrier, N. - Handbook of social functioning in Schizophrenia. Boston, Allyn and Bacon, 1997.

27. Bond, GR, Drake, RE y Becker, D R - «The role of social functioning in vocational rehabilitation». En (18) Mueser y Tarrier, 1998.:372-390.

28. Rosen, A y Barfoot, K.- "Day care and occupation: structured rehabilitation and recovery programmes and work». En Thornikroft, G y Szmukler, G. - Textbook of Community Psychiatry, Oxford University Press, 2001: 295-308.

29. Bell, M D, Lysaker, P H y Milstein, R M. - «Clinical benfits of paid work activity in Schizophrenia». Schizophrena Bulletin, 1996,22, 1: 51-67.

30. Bryson, G, Lysaker, P y Bell, M.- "Quality of life benefits of paid work activity in Schizophrenia». Schizophrenia Bulletin, 2002, 28, 2: 249-257.

31. Torrey, WC; Mueser, KT y ots.- "Self-esteem as an outcome measure in studies of vocational rehabilitation for adults with severe mental illness». Psychiatric Services, 2000, 51, 2:229-233.

32. Shepherd, G. - "The value of work in the 1980's». Psychiatric Bulletin, 1989, 13:231-233.

33. Goergen, R.- «Empresas y empresarios sociales en Italia: la experiencia de Trieste». En Lara y López, Op. Cit.,2003:158-200.

34. Lehman, A. F; Goldberg, R y ots. - «Improving employment outcomes for persons with severe mental illnesses». Archives of General Psychiatry, 2002,59, 2: 165-172.

35. Cook, J y Razzano, L - «Vocational rehabilitation for persons with Schizophrenia: recent research and implications for practice». Schizophrenia Bulletin, 2000, 26,1: 87-103.

36. Bond, GR, Drake, R E y ots.- «An update on supported employement for people with severe mental illness». Psychiatric Services, 1997,48: 336-346.

37. McGurrin, M C..- «An overview of the effectivenes of traditional vocational rehabilitation services in the treatment of long term mental illness». Psychosocial Rehabilitation J ournal, 1994,17,3:37-65.

38. Rutman, I D. - «How psychiatric disability expresses itself as a barrier to employement». Psychosocial Rehabilitation J ournal, 1994, 17,3:15-35.

39. Peckham, J. y Muller, J. (1999).- «Employment and Schizophrenia: recommendations to improve employability for individuals with schizophrenia». Psychiatric Rehabilitation J ournal, 22, 4: 399-402.

40. Aldaz, J A y Vázquez, C.- Esquizofrenia: fundamentos psicológicos y psiquiátricos de la rehabilitación. Madrid, Siglo XXI, 1996.

41. Ancil, R. J.,, Holliday, S. y Higgenbttam, J. (Ed.).- Schizophrenia. Exploring the spectrum of psychosis. Chichester, Wiley and Sons, 1994. 
42. Wing, J. - «Esquizofrenia». En Watts, F y Bennet, D. - Teoría y práctica de la rehabilitación psiquiátrica. México, Limusa, 1992:59-77.

43. Anthony, A W. - «Characteristics of people with psychiatric disabilities that are predictive of entry into the rehabilitation process and successful employement». Psychosocial Rehabilitation J ournal, 1994,17, 3: 3-13.

44. Turton, N.- «Welfare benefits and work disincentives». J ournal of Mental Health, 2001,10,3:285-300.

45. Warner, R.- «Work disincentives in US disability pension programs». J ournal of Mental Health, 2001,10,4:405-409.

46. Manning, Cy Withe, PD.- «Attitudes of employers to the mentally ill». Psychiatric Bulletin, 1995,19:541-543.

47. Simmons, TJ, Selleck, y ots. - «Supports and rehabilitation for employment». En Flexer, RW y Solomon, PL.- Psychiatric Rehabilitation in practice. Boston, Andover, 1993: 119-135.

48. Harding, C M y Zahniser, J H. - «Empirical correction of seven myths about schizophrenia with implications for treatment». Acta Psychiatrica Scandinavica., 1994, 90 (Supplem. 384): 140-146.

49. Anthony, WA y Blanch, A.- "Supported employment for persons who are psychiatrically disabled : an historical and conceptual perspective». Psychosocial Rehabilitation J ournal, 1987, 11:5-23.

50. O'Flynn, D y Craig, T.- «Which way to work? Occupations, vocations and opportunities for mental health service users». J ournal of Mental Health, 2001,10,1:1-4.

51. Bond, G.- «La integración laboral de personas con trastorno mental severo en Estados Unidos». En Lara y López, Op. Cit.,2003: 103-114.

52. Grove, B.- «La integración laboral de personas con trastorno mental severo en el Reino Unido». En Lara y López, Op. Cit.,2003:137-149.

53. Bond, G R.- «Applying Psychiatric Rehabilitation principles to employment: recent findings». En (24), Ancil, R y ots, 1994:49-65.

54. Bond, GR.- «Principles of the Individual Placement and Support model: empirical support». Psychiatric Rehabilitation J ournal, 1998, 22: 11-23. Traducción castellana en Lara y López, Op. Cit., 2003: 115-136.

55. Bond, G. R.,, Becker, D. R. y ots.- «l mplementing Supported Employment as an Evidence-Based Practice». Psychiatric Services, 2001,52 (3): 313-322.

56. Shepherd, G.- «Integración laboral de personas con enfermedad mental severa. Perspectiva nacional y local». En Lara y López, Op. Cit., 2003.

57. Reker, Th., Hornung, P. y ots. - «Long-term psychiatric patients in vocational rehabilitation programmes: a naturalistic follow-up study over 3 years». Acta Psychiatrica Scandinavica, 2000, 101:457-463. 
58. Latimer, EA.- «Economic impacts of supported employment for persons with severe mental illness». Canadian J ournal of Psychiatry, 2001,46:496-505.

59. Becker, D R, Smith, J . y ots. - «Fidelity of Supported Employment Programs and employment outcomes». Psychiatric Services, 2001,52, 6: 834-836.

60. Marrone, J., Balzell, A. y Gold, M. (1995).- «Employment supports for people with Mental IIIness». Psychiatric Services, 46,7: 707-711.

61. Banks, B; Charleston, S y ots.- «Workplace supports, job performance, and integration outcomes for people with psychiatric disabilities». Psychiatric Rehabilitation J ournal, 2001, 24,4:389-396.

62. Dorio, J; Guitar, A y ots.- «Differences in job retention in a supported employment program Chinook Clubhouse». Psychiatric Rehabilitation Journal, 2002, 25,3: 289-298.

63. Drake, RE; McHugo, GJ y ots.- «A randomized clinical trial of supported employment for inner-city patients with sever mental disorders». Archives of General Psychiatry, 1999, 56:627-633.

64. Henry, AD; Barreira, $P$ y ots. - «A retrospective study of Clubhouse-based transitional employment». Psychiatric Rehabilitation J ournal, 2001, 24,4:344354.

65. McDonald-Wilson, KL; Rogers, S y ots. - «An investigation of reasonable workplace accommodations for people with psychiatric disabilities: quantitative findings from a multi-site study». Community Mental Health J ournal, 2002,38,1: 35-50.

66. Furlong, M; McCoy, ML y ots. - "J obs for people with the most severe psychiatric disorders: Thresholds Bridge North Pilot». Psychiatric Rehabilitation J ournal, 2002, 26,1:13-22.

67. Gowdy, EL; Carlson, LS y Rapp, CA.- «Practices differentiating high-performing from low-performing supported employment programs». Psychiatric Rehabilitation J ournal, 2003, 26,3:232-239.

68. Wallace, CJ, Tauber, R y Wilde, J. (1999).- «Teaching fundamental workplace skills to persons with Serious mental IIIness». Psychiatric Services, 50,9:11471153.

69. Gallio, G. - Nell'I mpresa Sociale. Trieste, Per la salute mentale,1987.

70. Grove, B, Freudenberg, M y ots. - The social firm handbook. Brighton, Pavilion, 1997.

71. Schwarz, G. - «Las empresas sociales como medio para la integración laboral de personas con trastorno mental severo en Alemania.» En Lara y López, Op. Cit.,2003: 150-157.

72. Colón, C.- «La Fageda: una alternativa laboral para los enfermos mentales crónicos». Informaciones Psiquiatricas, 1992,131: 107-108.

73. Krupa, T; Lagarde, M y Carmichael, K.- « Transforming sheltered workshops into affirmative businesses: an outcome evaluation». Psychiatric Rehabilitation J ournal, 2003, 26,4:359-367. 
74. Manning, SS y Suire, B.- «Consumers as employees in Mental health: bridges and roadblocks». Psychiatric Services, 1996,47:939-940.

75. Bedia, M., Arrieta, M. y ots.- "Rehabilitación sociolaboral de personas con esquizofrenia: variables relacionadas con el proceso de inserción». Actas Españolas de Psiquiatría., 2001,29, 6:357-367.

76. Jacobs, H E, Wissusik, D y ots (1992).- «Correlations between psychiatric disabilities and vocational outcome». Hospital and Community Psychiatry,43, 4: 365-369.

77. Anthony, W A, Rogers, E S y ots.- «Relationship between psychiatric symptomatology, work skills, and future vocational performance». Psychiatric Services, 1995,46, 4: 353-358.

78. Lysaker, P H, Bell, M D y ots. - «Social skills at work. Deficits and predictors of improvement in schizophrenia». The Journal of Nervous and Mental Disease, 1995, 183, 11: 688-692.

79. Lysaker, P H, Bell, M D y ots. - «Work capacity in Schizophrenia». Hospital and Community Psychiatry, 1993,44: 278-280.

80. Rogers, S; Anthony, WA y ots.- "Prediction of vocational outcome based on clinical and demographic indicators among vocationally ready clients». Community Mental Health journal, 1997,33,2:99-112.

81. Suslow, $\mathrm{T}$; Schonauer $\mathrm{K}$ y ots.- «Prediction of work performance by clinical symptoms and cognitive skills in schizophrenic outpatients». J ournal of Nervous and Mental Diseasses, 2000,188,2:116-118.

82. J ones, Cl; Perkins, D y Born, DL.- «Predicting work outcomes and service use in supported employment services for persons with psychiatric disabilities». Psychiatric Rehabilitation J ournal, 2001, 25,1:53-59.

83. Bell, M D y Bryson, G.- «Work rehabilitation in Schizophrenia: Does cognitive impairment limit improvement?». Schizophrenia Bulletin, 2001, 27 (2): 269-279.

84. Gold, J M, Goldberg, R W, y ots. - «Cognitive correlates of job tenure among patients with severe mental illness». American J ournal of Psychiatry, 2002,159: 1395-1402.

85. Green, M F.- «What are the functional consequences of neurocognitive deficits in schizophrenia?». American J ournal of Psychiatry, 1996,153: 321-330.

86. Mueser, K T, Becker, D R y ots. - « Work and nonvocational domains of functioning in persons with severe mental illness: a longitudinal analysis». The J ournal of Nervous and Mental Disease, 1997,185, 7: 419-426.

87. Velligan, DI, Bow-Thomas, C C, y ots -- «Do specific neurocognitive deficits predict specific domains of community function in Schizophrenia?». The J ournal of Nervous and Mental Disease, 2000,188, 8: 518-524.

88. Liberman, R P y Green, M F. - «Wither cognitive-behavioral therapy for schizophrenia». Schizophrenia Bulletin, 1992, 18, 1: 27-35. 
89. Mueser, K T, Bond, G R y Drake, R. - " Community-based treatment of Schizophrenia and other Severe Mental disorders: Treatment outcomes?». Medscape Mental Health, 6 (1) (En www.medscape.com). Traducción castellana en Lara y López, Op. Cit., 2003: 26-64.

90. Provencher, HL; Gregg, R y ots.- "The role of work in the recovery of persons with psychiatric disabilities». Psychiatric Rehabilitation J ournal, 2002, 26,2:132144.

91. Cunningham, K, Wolbert, R y Brockmeier, M B.- «Moving beyond the illness: factors contributing to gaining and maintaining employment». American J ournal of Community Psychology, 2000,28, 4: 481-494.

92. Mueser, KT; Becker, D y Wolfe, R.- "Supported employment, job preferences, job tenure and satisfaction». J ournal of Mental Health, 2001,10,4:411-417.

93. Casper, ES y Fishbein, S. - « ob satisfaction and job success as moderators of the self-esteem of people with mental illness». Psychiatric Rehabilitation J ournal, 2002, 26,3:33-42.

94. Rollins, AL; Mueser, KT y ots.- «Social relationships at work: does the employment model make a difference?». Psychiatric Rehabilitation J ournal, 2002, 26,1:5161.

95. Kravetz, S; Dellario, D y ots.- "A two-faceted participation approach to employment and career development as applied to persons with a Psychiatric disability». Psychiatric Rehabilitation J ournal, 2003, 26,3:278-289.

96. Holzner, B, Kemmler, G y Meise, U.- «The impact of work-related rehabilitation on the quality of life of patients with Schizophrenia». Social Psychiatry and Psychiatric Epidemiology, 1998,33: 624-631.

97. Lal, S y Mercier, C.- "Thinking out of the box: an intersectorial model for vocational rehabilitation». Psychiatric Rehabilitation J ournal, 2002, 26,2:145153.

98. López, M.- «Situación actual y perspectivas del programa de integración laboral de personas con trastorno mental severo en Andalucía». Boletín de la Asociación Madrileña de rehabilitación Psicosocial, 2001,8,13: 51-58.

99. Hervás, A; Cuevas, C y ots.- «Estudio descriptivo de la situación laboral de la población con trastorno esquizofrénico del Area Sanitaria «Virgen del Rocío» de Sevilla». Intervención Psicosocial, 1997, VI,3:393-404.

100. Comunidad de Madrid.- Rehabilitación laboral de personas con enfermedad mental crónica: Programas básicos de intervención. Cuadernos Técnicos de Servicios Sociales, ํㅡ 14, 2001.

101. Rodríguez, A.- «La experiencia de los Centros de Rehabilitación Laboral del Programa de Atención social a personas con enfermedad mental crónica de la Consejería de Servicios Sociales de la Comunidad de Madrid». En Lara y López, Op. Cit., 2003:210-228. 
102. Alvarez, F.- «Programas de integración laboral de personas con trastorno mental severo en Andalucía». En Lara y López, Op. Cit., 2003:201-209.

103. Saraceno, B , De Luca, L y Montero, F. (1992).- «Problemas metodológicos en la evaluación de un programa de rehabilitación». Revista de la Asociación Española de Neuropsiquiatría, 1992, XII, 40: 3-8.

104. Phillips, C, Palfrey, C y Thomas, P. - Evaluating Health and Social Care. London, Macmilllan, 1994.

105. Hollannd, W W (Ed).- Evaluation of Health Care. Oxford University Press, 1983.

106. WHO. - Health Program Evaluation. Geneva, WHO, 1981.

107. Cochrane, A L. - Eficacia y eficiencia. Reflexiones al azar sobre los servicios sanitarios. Barcelona, Salvat, 1985.

108. Aparicio, V. (Coord.).- Evaluación de servicios de salud mental. Madrid, AEN, 1993.

109. Amezcua, C. y J iménez, A. - Evaluación de programas sociales. Madrid, Díaz de Santos, 1996.

110. Donabedian, A.- La calidad de la atención médica. Definición y métodos de evaluación. México DF, Prensa Médica Mexicana, 1984.

111. Boothroyd, R A, Evans, M E y ots. - "Quality Assurance and Evaluation of Psychiatric rehabilitation programs». En Flexer, RW y Solomon, PL (Ed).Psychiatric rehabilitation in practice. Stoneham, Andover, 1993: 211- 227.

112. Thornicroft, Gy Bebbington, P. - «Quantitative methods in the evaluation of Community Mental Health Services». En Breakey, WR.- Integrated Mental Health Services. Modern Community Psychiatry. New York, Oxford University Press, 1996: 120-138.

113. Tsuang, M Ty Tohen, M (Ed) .- Textbook in Psychiatric Epidemiology. 2nd. Edition, New York, Wiley-Liss, 2002.

114. Asociación Española de Neuropsiquiatría.- Rehabilitación psicosocial del trastorno mental severo. Situación actual y recomendaciones. Madrid, AEN, 2002, Cuadernos Técnicos, $\mathrm{n} 06$.

115. Salvador-Carulla, L, Bulbena, A y ots.- «La Salud Mental en España: Cenicienta en el País de las Maravillas». En Informe SESPAS 2002. Invertir en salud. .Prioridades en Salud pública. Valencia, EVES, 2002:301-326. 
López Alvarez, Marcelino

Psiquiatra, Sociólogo. Director de programas, evaluación e investigación. FAISEM. Sevilla

Laviana Cuetos, Margarita

Psicóloga Clínica. Coordinadora de la Comunidad Terapéutica del Area de Salud Mental del Hospital «Virgen del Rocío». SAS. Sevilla

Alvarez J iménez, Francisco

Economista. Gerente de IDEA, SA. Sevilla

González Alvarez, Sergio

Psicólogo. Técnico de FAISEM. Sevilla

Fernández Doménech, María

Terapeuta Ocupacional. Técnica de FAI SEM. Sevilla

Vera Peláez, Maria Paz

Trabajadora Social. Técnica de IDEA, SA. Sevilla

Correspondencia:

Marcelino López Alvarez. Fundación Andaluza para la Integración Social del Enfermo mental. Avenida de las Ciencias, 27, Acceso A. 42020. Sevilla.

marcelino.lopez@juntadeandalucia.es

La revisión bibliográfíca que sirve de base al articulo se ha realizado en el marco del proyecto de investigación «Evaluación de los programas de integración laboral para personas con trastorno mental severo en Andalucía», financiado por el FIS (Exp. PI021264). 